\title{
Transient global amnesia associated with migraine triggered by anxiety under the effects of the coronavirus (COVID-19) pandemic:A case report
}

\begin{abstract}
Transient global amnesia (TGA) is a clinical syndrome featured with the sudden onset of primarily short-term loss of anterograde as well as a milder decline of retrograde memory. The etiology is still unclear. Various risk factors relate with TGA and it is thought the vulnerability of CA1 neurons to metabolic stress plays an important role in the pathophysiological cascade. During the quarantine period of the coronavirus (COVID-19) pandemic, a 53-year-old Asian woman with 30 years of migraine history presented the emergency department for the first time to evaluate a sudden onset confusion and forgetfulness with repetitive questioning during migraine attack. Neurologic examination showed preserved orientations for time and person and no abnormalities in motor, speech, sensory, coordination, or cranial nerves. No focal Neurologic finding. Her memory gradually improved and restored to normal baseline over the course of a 24-hour in-patient stay. However, are trograde memory gap still existed a month after the TGA attack. The pathogenesis of TGA is unknown and many risk factors are associated with it, but among them migraine is considered a major risk factor, particularly in female patients aged 40-60 years. The anxiety stressor is a significant trigger for TGA. The pathophysiology argues that the vulnerability of CA1 neurons to metabolic stress plays an important role in TGA.
\end{abstract}

Volume 10 Issue 3 - 2020

\author{
Chunhui Yang,' Junyi Zhang, ${ }^{2}$ Sandeep \\ Gaonkar' \\ 'Conventions Psychiatry \& Counseling, USA \\ ${ }^{2}$ Neurology department, People's Hospital of Ordos Dongsheng \\ District, China
}

Correspondence: Chunhui Yang, Conventions Psychiatry \& Counseling, I560 Wall St. Naperville, IL 60563, USA,

Email Chunhuiyang2018@gmail.com

Received: May 31, 2020 | Published: June 12, 2020

Keywords: Transient global amnesia (TGA), migraine, amnesia, anterograde memory, short term memory, COVID-19 pandemic

\section{Introduction}

Transient global amnesia (TGA), a reversible and relatively shortterm amnesic syndrome of sudden onset that is sometimes associated with migraine. TGA shows predominantly anterograde amnesia as well as a weaker retrograde amnesia with returning to normal baseline around 24 hours. Generally, the patients display no other signs of impaired cognitive functioning and present no focal neurologic deficits. ${ }^{1,2}$ In 1956, an "isolated episode of confusion with amnesia" was described. The term TGA was created by Fisher and $\mathrm{Adams}^{3}$ in 1958. Nevertheless, at present this syndrome continues to be one of the most inexplicable syndromes in clinical neurology.

The fundamental pathophysiology of TGA has not been completely elucidated. Multiple advocated mechanisms, such as arterial ischemia, migraine, venous congestion, and psychogenic disorders have been raised. ${ }^{4,5}$ Among them, the migraine is considered as a remarkable risk factor associated with emerging TGA. ${ }^{6-9}$ It has been identified the disruption of the neurons in CA1 of the hippocampus plays a crucial role in the pathophysiology of TGA due to their distinct sensitivity to stress attack. ${ }^{10}$

\section{Case report}

A 53-year-old Asian female presented to the emergency department with a sudden onset of confusion and forgetfulness. The patient stated she had headache for about 10 hours. The patient said she had migraine history for almost 30 years. Her migraine was a regular occurrence once a few months. However, during the period of the quarantine of the coronavirus (COVID-19) pandemic, her migraine attacks were more frequent and severe than before because of the extreme stress of worrying about losing the job. This time, though, she took the Zomig as usual, but it did not work. Per the patient's husband, in the late afternoon, while he was massaging the patient's head and neck to relieve the headache, he noticed she became confused, disoriented, and she was repeatedly asking the same questions. The patient had never experienced the similar episodes before. The patient vomited two times. The patient did not have any visual auras before the migraine onset. The patient denied any strange smell, taste, or any sensations of depersonalization. The patient had no loss of consciousness, vertigo, double vision, numbness, weakness or garbled speech. The patient denied fever, chest pain, shortness of breath, abdominal pain, or any changes in bowel or urinary movement. The patient's past medical history was significant only for migraine without any seizure or psychiatric disorders. The patient was taking Zomig $(2.5 \mathrm{mg})$ as needed. The patient had no history of allergies to any drugs. The patient had no history of any narcotic drugs. The patient was an accountant and was married, living with her husband and one child. The patient carried no family history of any neurologic disease or psychiatric disorders. The vital signs were normal with the blood pressure of $136 / 84 \mathrm{~mm} \mathrm{Hg}$, the heart rate of 75 beats/minute, and the temperature of $98.2^{\circ} \mathrm{F}$. The patient looked well-developed and well-nourished without any distress. There were no carotid bruits in the neck. The examination of the cardiovascular, lungs, and abdomen revealed no significant abnormalities. 
Neurological examination showed undamaged mental status. The patient was alert, and oriented to person, place, and time. She knew who she was. She could recognize the family members. She was constantly asking the same questions such as "what happened?". Longterm memory was intact (She could remember her birthday) and she was conscious of current events, but obviously her short-term memory was compromised. A significant anterograde amnesia was found, for example, she even forgot about her daughter getting into high school a few months ago. There were no hallucinations or delusions existed. She could easily recognize the family members. Her speech was fluent. All the cranial nerve exhibited intact. No nystagmus was seen. The motor examination was intact. No significant abnormalities were found in coordination, sensation, and gait. The Babinski sign was not seen. The Kernig's sign was negative. The results of the complete blood count, the chemistry panel, the thyroid stimulating hormone, the coagulation profile and the urinalysis were all in normal ranges. The electrocardiogram showed sinus rhythm, and the chest X-ray was normal. Head CT (computed tomography) revealed no acute bleeding. The head DWI (diffusion-weighted imaging), MRI (Magnetic resonance imaging) and the MRA (magnetic resonance angiogram) discovered no significant abnormalities. The bilateral carotid duplex showed no atherosclerotic plaques in the internal carotid arteries. An EEG (electroencephalogram) exam was also normal. Lumbar puncture was performed and there were no abnormal findings in CSF. The patient's memory was gradually returning to the normal baseline in 24 hours when she was inpatient observation. However, at the two month following up, a retrograde amnesic gap for the on-going events during the TGA attack still existed. The patient said she could only recall a few intermittent voice conversations, but all the relevant pictures were black.

\section{Discussion}

The key clinical features of the TGA are the abrupt onset and the interruption of the short-term memory, while the other cognitive functioning is intact. ${ }^{11,12}$ The etiology of TGA remains unknown. A migraine history is considered as one of the more prominent risk factors related to the development of the TGA. ${ }^{7}$ For most of the patients with migraine, TGA generally occurred after the onset of migraine. ${ }^{8,13}$ It has been identified that TGA occurs in certain backgrounds. Some precipitating factors for TGA, including emotional stress, were emphasized. Anxiety produced by tension in daily life from work, health problems, and financial stressors were often reported weeks prior to TGA and are considered "remote events". ${ }^{14-20}$ Our patient had a migraine history for 30 years and this was the first time for TGA onset in the special period of the pandemic. The likely unfavourable effects of the COVID-19 pandemic on people might be aggravated by fear, self-isolation, and physical distancing. The patient acknowledged that she was worrying about of losing her job and fearing of her family's finances. All these stresses put tremendous pressure on her.

It has been reported that one-third of TGA episodes occur after physical or psychological tensions. This phenomenon suggested disruption of memory formation due to stress-induced catecholamine disruption. ${ }^{17,18,20}$ Some studies indicated the deterioration of the neurons in CA1 sector of the hippocampus under the enormous glutamate release in the state of severe emotional effects and migraines. The experiment using the animal models through local stimulation of the hippocampus, observing a similar reaction of producing a large release of glutamate from hippocampus, as that seen in humans via the experience of strong emotional events or other intense stimuli. ${ }^{19}$ Physiologically, the affective learning circuit formed between amygdala, hippocampus, striatum, and prefrontal cortex, is disrupted by such events. TGA could be assessed as an affliction of temporary hippocampal deficiency, in which its inhibitory effects to the amygdala are disturbed, to cause a disruption in memory formation. ${ }^{14}$ These evidences definite the relationship between migraines and transient hippocampal dysfunction. It is acceptable to consider the likelihood of an etiological association between migraines and the transient memory problems demonstrated by patients with TGA. ${ }^{17}$

Although the physiological findings suggest a meaningful association between a venous congestion and TGA, there is limited evidence for it as an etiology for TGA. ${ }^{21-23}$ The TGA in our patient was onset in the moment when her husband was massaging her head and neck for relieving the headache, which raised the possibility that the massaging might related to the TGA happening.

Our patient met all the diagnostic criteria: (1) exclude head trauma or loss of consciousness at the onset; (2) no accompanying focal neurologic signs or symptoms; (3) the memory loss resolved around 24hours; (4) No epileptic features and no active epilepsy existed. The arterial ischemia is believed as one etiology for TGA due to TIA sharing common features as TGA, such as returning to normal less than 24hours; However, TGA episodes usually last longer than TIA. It was most reliably seen returning to normal around 24-72hours after symptom onset and some of the patients had the permanent sequence of amnesia gap after TGA onset.

TGA also requires to be distinguished with psychogenic amnesia ${ }^{9}$ which often characterized as dissociative amnesia. Psychogenic amnesia often primarily affects retrograde memory. Our patient mainly showed anterograde amnesia and did not carry any psychiatric disorders history.

The amnesia caused by TEA (temporal epilepsy amnesia) may appear antero-retrograde or only retrograde. ${ }^{21}$ While amnesia from TGA primarily presents anterograde memory loss and may have permanent retrograde amnesia gap.

Consistent with the standard accepted criteria, the symptoms of TGA can gradually resolves without mediation. ${ }^{16}$ The studies have shown that after TGA onset, for the 30-day period following up, there was no significant difference between patients and controls in any of the anterograde memory, retrograde memory, short-term memory, semantic memory, and executive function. ${ }^{15}$ Our patient still showed amnesic gap at the 2-month follow-up visit, which consistent with the report ${ }^{2}$ that most patients with TGA had a permanent retrograde amnesic gap for the events around the TGA attack. Our patient was able to recall several audio-memory recollections during TGA events, but all the relevant plot images associated with them were black. This phenomenon confirmed a different route for audio and graphic memory.

Although the recurrence rate of TGA is less $6.3 \%$, an increased risk of mortality, epilepsy, or stroke following TGA is rarely seen..$^{22}$ Nevertheless, it is extreme necessary to provide the appropriate intervention to the patients with migraine to prevent from TGA attack, such as learning relaxation techniques and taking proper medication to prevent migraines.

We reported a case of a middle-aged woman with a 30-year history of migraine headache that worsened during the COVID-19 pandemic due to high anxiety and a TGA was sudden onset after the migraine attack. This case supported migraine is one of the main risk factors for TGA and the emotional stress can trigger it. 


\section{Conflicts of interest}

The authors have no conflicts of interest to declare.

\section{Funding}

None.

\section{Acknowledgments}

None.

\section{References}

1. David Gunnell, Louis Appleby, Ella Arensman, et al. Suicide risk and prevention during the COVID-19 pandemic. The Lancet psychiatry. 2020;7(6):468-471.

2. Hodges JR, Warlow CP. Syndromes of transient amnesia: towards a classification. A study of 153 cases. J Neurol Neurosurg Psychiatry. 1990;53(10):834-843.

3. Fisher CM, Adams RD. Transient global amnesia. Trans Am Neurol Assoc. 1979;104:183-186.

4. Jang JW, Park SY, Hong JH, et al. Different risk factor profiles between transient global amnesia and transient ischemic attack: a large casecontrol study. Eur Neurol. 2014;71(1-2):19-24.

5. Lee HY, Kim JH, Weon YC, et al. Diffusion-weighted imaging in transient global amnesia exposes the CA1 region of the hippocampus. Neuroradiology. 2007;49(6):481-487.

6. Yang Y, Kim S, Kim JH. Ischemic evidence of transient global amnesia: location of the lesion in the hippocampus. J Clin Neurol. 2008;4(2):59-66.

7. Miniju Yi, Ayesha Z. Sherzai, et al. Strong Association Between Migraine and Transient Global Amnesia: A National Inpatient Sample Analysis. The Journal of Neuropsychiatry and clinical Neuroscience. 2018; 31(1):4348.

8. Arena JE, Brown RD, Mandrekar J, et al. Long-term outcome in patients with transient global amnesia: a population-based study. Mayo Clin Proc. 2017;92(3):399-405.

9. Inzitari D, Pantoni L, Lamassa M, et al. Emotional arousal and phobia in transient global amnesia. Arch Neurol. 1997;54(7):866-873.

10. Lee HY, Kim JH, Weon YC, et al. Diffusion-weighted imaging in transient global amnesia exposes the CA1 region of the hippocampus. Neuroradiology. 2007;49(6):481-487.
11. Sancesario G, Esposito Z, Mozzi AF, et al. Transient global amnesia: linked to a systemic disorder of amino acid catabolism? J Neurol. 2013;260(5):1429-1432.

12. Alemdar M, Selekler M. Migraine and cortical spreading depression. Agri. 2006;18(4):24-30.

13. Donnet A. Transient global amnesia triggered by migraine in a French Tertiary-Care Center: an 11-year retrospective analysis. Headache. 2015;55(6):853-859.

14. Spiegel DR, Mccroskey AL, Deyerle BA. A case of transient global amnesia: a review and how it may shed further insight into the neurobiology of delusions. Innov Clin Neurosci. 2016;13(3-4):32-41.

15. Jäger T, Bäzner H, Kliegel M, et al. The transience and nature of cognitive impairments in transient global amnesia: a meta-analysis. $J$ Clin Exp Neuropsychol. 2009;31(1):8-19.

16. Hodges JR, Warlow CP. Syndromes of transient amnesia: towards a classification. A study of 153 cases. J Neurol Neurosurg Psychiatry. 1990;53(10):834-843.

17. Fisher CM. Transient global amnesia. Precipitating activities and other observations. Arch Neurol. 1982;39(10):605-608.

18. Miller JW, Petersen RC, Metter EJ, et al. Transient global amnesia: clinical characteristics and prognosis. Neurology. 1987;37(5):733-737.

19. Olesen J, Jørgensen MB. Leao's spreading depression in the hippocampus explains transient global amnesia: a hypothesis. Acta Neurol Scand. 1986;73(2):219-220.

20. Inzitari D, Pantoni L, Lamassa M, et al. Emotional arousal and phobia in transient global amnesia. Arch Neurol. 1997;54(7):866-873.

21. Kapur N. Transient epileptic amnesia-a clinical update and a reformulation. J Neurol Neurosurg Psychiatry. 1993;56(11):1184-1190.

22. Quinette P, Guillery-Girard B, Dayan J, et al. What does transient global amnesia really mean? Review of the literature and thorough study of 142 cases. Brain. 2006;129(pt 7):1640-1658.

23. Chung CP, Hsu HY, Chao AC, et al. Transient global amnesia: cerebral venous outflow impairment - insight from the abnormal flow patterns of the internal jugular vein. Ultrasound Med Biol. 2007;33(11):1727-1735. 